Original Research Article

\title{
Comparative evaluation of efficacy of terbinafine and itraconazole in treatment of tinea cruris
}

\author{
Monica George ${ }^{1}$, Raju G. Chaudhary ${ }^{2 *}$, Devang Rana ${ }^{3}$, Dixit Kasundra ${ }^{2}$, \\ Abhishek R. Chaudhary ${ }^{4}$, Supriya D. Malhotra ${ }^{3}$
}

\author{
${ }^{1}$ Student, Smt. NHL Muncipal \\ Medical College, Ahmedabad, \\ Gujarat, India \\ ${ }^{2}$ Department of Dermatology, \\ V.S. Hospital, Ahmedabad, \\ Gujarat, India \\ ${ }^{3}$ Department of Pharmacology, \\ Smt. NHL Muncipal Medical \\ College, Ahmedabad, Gujarat, \\ India \\ ${ }^{4}$ Student, GMERS Medical \\ College, Sola, Gujarat, India
}

Received: 15 May 2019

Accepted: 10 June 2019

*Correspondence to:

Dr. Raju G. Chaudhary,

Email: abhijeetraju@

yahoo.co.in

Copyright: (C) the author(s), publisher and licensee Medip Academy. This is an openaccess article distributed under the terms of the Creative Commons Attribution NonCommercial License, which permits unrestricted noncommercial use, distribution, and reproduction in any medium, provided the original work is properly cited.

\begin{abstract}
Background: In a hot and humid country like India, the prevalence of superficial mycotic infections is on the rise due to contributing environmental and demographic factors. In this study, we sought to assess the efficacies of two oral antifungal drugs, Itraconazole (a traditional azole) and Terbinafine (the only orally available allylamine). The two drugs were analyzed to see whether they differed significantly in their cure rates of tinea cruris. Since data, that compares only systemically administered Itraconazole and Terbinafine in the treatment of tinea cruris, is limited, this study becomes imperative.

Methods: 60 patients, all clinically confirmed cases of tinea cruris and belonging to the age group of 18-65 years, were recruited for this prospective study. Patients were then randomly divided into groups A and B and followed-up for a month. Group A received cap. Itraconazole $100 \mathrm{mg}$, twice a day, for 15 days while group $\mathrm{B}$ received tab. Terbinafine $250 \mathrm{mg}$, once a day, for 4 weeks. Both groups were given topical $2 \%$ Sertaconazole that had to be continued for 8 weeks. During the 4 visits, scores for the 3 parameters (erythema, pruritis and scaling) were calculated and recorded for statistical analysis.

Results: Authors observed that majority of the patients were exposed to hot and humid environment that contributed to sweating and poor personal hygiene. The changes in scores of erythema, pruritis and scaling from the baseline visit for both, Itraconazole and Terbinafine, were statistically significant at week 4 with $\mathrm{P}$ $<0.05$ for all parameters. But the difference between the scores of the two drugs was not found to be statistically significant.

Conclusions: Although the sample size of this study was small and the data was limited, findings of this study supported that both Itraconazole and Terbinafine were highly effective in the treatment of tinea cruris.
\end{abstract}

Keywords: Efficacy, Itraconazole, Terbinafine, Tinea Cruris

\section{INTRODUCTION}

Superficial mycosis is estimated to be one of the most prevalence infections, affecting roughly quarter of the world's population. ${ }^{1}$ Among the various classes of cutaneous mycoses, dermatophytes are the most common causative agents. Generally referred to as ringworm or tinea, this specific group of dermatophytes include the genera Microsporum, Trichophyton and Epidermophyton. Transmission of this disease occurs either by direct contact 
with infected humans or animal or indirectly by contact with contaminated fomites. ${ }^{2}$ Incidence of dermatophytosis in India ranges from $36.6 \%$ to $78.4 \%$ due to the high temperature and humidity that contributes to excess sweating, maceration, and alkaline $\mathrm{pH}$; these environmental factors are ideal for the keratinophilic fungi. ${ }^{3,4}$ Hence, certain parts of the body, namely intertriginous areas (web spaces and groins) are more susceptible to the infections since they provide favorable media for the fungi to grow..$^{5}$ Despite being a superficial and largely painless, tinea can also invade deeper into the tissues and cause a disseminated infection, depending on the host's immune status and hence the disease should not be neglected. ${ }^{6}$ The presentations and severity of tinea is dependent largely on the host's immune status. Diseases such as diabetes mellitus, lymphomas, Cushing's syndrome as well as old age compromise a person's immunity and can result in severe and widespread dermatophytosis. ${ }^{4}$

True to its more common name, ringworm, this cutaneous fungal infection has a classical appearance of a central clearing that is surrounded by an active border of redness and scaling. ${ }^{7}$ When present in the inguinal region, this disease presents as bilateral, dull red, pruritic plaques that have prominent scaly edges and minute papules or pustules at their margins. ${ }^{8,9}$

Traditionally, azole antifungals such as Itraconazole, when present in therapeutic concentrations, interrupt the functions of ergosterol that is present in the fungal membranes and disrupt both the structure of the membrane as well as its functions (nutrient transport and chitin synthesis). ${ }^{10}$ Sertaconazole, on the other hand, is a relatively new benzothiophene imidazole derivative that indirectly inhibits ergosterol synthesis and directly inhibits the non-sterol component of the fungal cell membrane as well. Its collective action results in leakage of intracellular components and results in rapid cell death. ${ }^{11}$ In addition, the lipophilic property of the benzothiophene ring enables prolonged dermal retention that permits just once-daily application of $2 \%$ sertaconazole cream. ${ }^{4}$

Terbinafine is the only orally administered allylamine that is available commercially. It inhibits ergosterol synthesis at the stage of squalene epoxidation and is highly effective for majority of the dermatophytes. Terbinafine demonstrates a good safety profile while achieving high concentrations in keratinous tissues that are maintained for up to 3 months. ${ }^{12}$

The antifungal agents utilized for this study were Terbinafine and Itraconazole, both of which were systemically administered, along with topical $2 \%$ Sertaconazole cream. We sought to assess whether two oral antifungal drugs, Itraconazole and Terbinafine differed significantly in their cure rates of tinea cruris in this study.
The objective of this study was to compare the therapeutic efficacy of Terbinafine and Itraconazole in the treatment of tinea cruris.

\section{METHODS}

It was a prospective, observational study conducted in the dermatology outpatient of a tertiary care, teaching hospital in Ahmedabad, Gujarat. Clinically confirmed cases of tinea cruris were recruited for the study and followed up for a month, till the completion of their treatment.

All consenting patients, in the age group of 18-65 years, who were diagnosed by the dermatologist as suffering from tinea cruris were included in the study, irrespective of the presence and extent of dermatophytosis in other regions of the body. Patients who were pregnant, lactating, non-consensual, as well as those who had a history of antimycotic treatment within a month prior to baseline visit were excluded from the study.

The study was conducted after obtaining the permission from institutional review board. After informed written consent, the patient's history (age, sex, family history, precipitating factors (diabetes, hypertension, poor hygiene), past history of infection and treatments, duration of current infection and associated disorders) was obtained and he/she was evaluated on the basis of three parameters: erythema, scaling and pruritis. Each parameter was graded on a 4 -point scale $(0=$ absent, $1=$ mild, $2=$ moderate and $3=$ severe). ${ }^{13}$ Erythema and scaling were scored by the dermatologist while pruritus was graded with a $10 \mathrm{~cm}$ long visual analog scale (Figure 1) under direct supervision of the dermatologist (Score 0 on the visual analog scale $=$ absent, scores $1-3=$ mild, $4-7=$ moderate and 8 $10=$ severe $)^{4,14}$

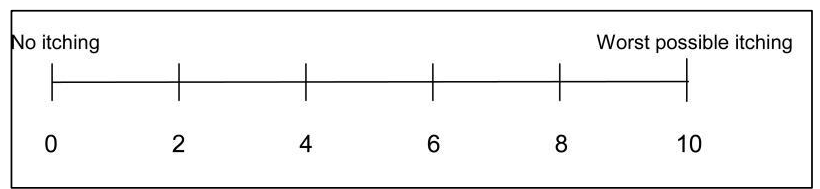

Figure 1: Visual analog scale.

Patients were then randomly divided into groups A and B and allotted the respective drugs (Table 1). They were followed-up every week for a month and during each visit, the scores were calculated as mentioned above and recorded for statistical analysis. Any reported adverse drug reactions were also noted.

\section{Statistical analysis}

Microsoft Excel 2013 and IBM SPSS Statistics (version 20) were used to analyse the data. The data attained was analysed on the basis of drug distribution as per age, sex, predisposing factors (diabetes, hypertension, poor hygiene) and recurrence. The changes in symptom score from baseline for each drug as well as the difference in the 
scores between the two drugs at the end of the $4^{\text {th }}$ week were analysed and assessed for statistical significance were also by using Student's t-test. $\mathrm{P}<0.05$ was considered statistically significant.

Table 1: Drugs prescribed.

\begin{tabular}{|ll|}
\hline Groups & Prescription \\
\hline A & $\begin{array}{l}\text { Cap. Itraconazole 100mg BD for } 15 \text { days } \\
\text { (along with topical } 2 \% \text { Sertaconazole for } 8 \\
\text { weeks). }\end{array}$ \\
\hline B & $\begin{array}{l}\text { Tab. Terbinafine 250mg OD for } 4 \text { weeks } \\
\text { (along with topical } 2 \% \text { Sertaconazole for } 8 \\
\text { weeks). }\end{array}$ \\
\hline
\end{tabular}

\section{RESULTS}

Out of the 60 consenting patients included in the study, 28 patients were prescribed Itraconazole (group A) and remaining 32 patients were prescribed Terbinafine (group B). The male-to-female ratio in this study was 1.069 .

The ages of the consenting patients varied vastly, ranging from 18 years of age to 77 years of age. Overall, 23 patients in the study belonged to the age group of 11-30 years. Majority of the patients in group A were in the age group 21-30 years of age whereas majority of the patients in group B belonged to 51-60 years of age. $97 \%$ of the patients who participated in this study belonged to lower socio-economic class. 45 patients claimed to not be suffering from any other diseases.

The most frequent co-morbidity in this study was hypertension ( 9 patients), followed by diabetes (8 patients). 3 patients in group A and 6 patients in group B suffered from high blood pressure. None of the patients in group A suffered from diabetes. 45 patients claimed to not be suffering from any other diseases. 19 patients in group A and 20 patients in group B admitted to practicing poor personal hygiene (skipping showers, not washing their hands, poor oral hygiene). 5 and 12 patients with a family history of tinea cruris (one or more of the patient's family members suffered from the infection in the past month or so) received Itraconazole and Terbinafine respectively. 17 patients in group $\mathrm{A}$ and 21 patients in group B complained of a prior history of the disease. 16 patients had used topical steroids month(s) priors to the baseline visit $(8,8$ in group A, B respectively).

There were 24 patients also complained of similar symptoms of itching and redness in other parts of the body (chest and back more frequently). One out of the 28 patients in group A discontinued his medication after the second week due to the lack of results. Similarly, one of the patients in group B discontinued his medication in the fourth week. Two patients, one from group A and the other from group B, complained of abdominal discomfort but continued the medications, nevertheless (Table 2).

Table 2: Frequency of the scores at baseline visit.

\begin{tabular}{|c|c|c|c|c|c|c|}
\hline \multirow{2}{*}{ Score } & \multicolumn{2}{|l|}{ Erythema } & \multicolumn{2}{|l|}{ Pruritis } & \multicolumn{2}{|l|}{ Scaling } \\
\hline & Group A & Group B & Group A & Group B & Group A & Group B \\
\hline 0 (absent) & 2 & 0 & 4 & 0 & 1 & 1 \\
\hline 1 (mild) & 7 & 13 & 10 & 11 & 3 & 17 \\
\hline 2 (moderate) & 15 & 14 & 12 & 14 & 10 & 13 \\
\hline 3 (severe) & 4 & 5 & 2 & 7 & 14 & 11 \\
\hline
\end{tabular}

Table 3: Frequency of the scores in the 4th week.

\begin{tabular}{|lllllll|}
\hline Score & Erythema & & Pruritis & & Scaling \\
& Group A & Group B & Group A & Group B & Group A & Group B \\
\hline 0 (absent) & 16 & 24 & 11 & 17 & 15 & 25 \\
\hline 1 (mild) & 11 & 7 & 14 & 14 & 12 & 5 \\
\hline 2 (moderate) & 0 & 0 & 2 & 0 & 0 & 1 \\
\hline 3 (severe) & 0 & 0 & 0 & 0 & 0 & 0 \\
\hline
\end{tabular}

During baseline visits, majority of patients in group A suffered from moderate erythema and pruritis but severe scaling was more frequently seen. Similar findings were evident for group B as well. Group B saw highest frequency of moderate scores in all three parameters (Table 3). During the $4^{\text {th }}$ visit, group B showed higher improvement rates as compared to group A. Group B had higher frequency of score 0 in all three parameters. There were 2 patients in group B who complained of moderate pruritis while one patient in group B complained of moderate scaling at the end of week 4 (Table 3).

The changes in erythema and scaling from baseline visit for group A (Itraconazole) were statistically significant at week 4 with $\mathrm{P}$ value being less than 0.0001 for both 
parameters. The change in pruritis from the baseline visit, too, was statistically significant at week 4 with $\mathrm{P}$ value equaling 0.0004 (Table 4). The changes in erythema, pruritis and scaling from the baseline visit for group B (Terbinafine) were statistically significant at week 4 with
$\mathrm{P}$ value being less than 0.0001 for all parameters. The changes in erythema, pruritis and scaling from the baseline visit for group B (Terbinafine) were statistically significant at week 4 with $\mathrm{P}$ value being less than 0.0001 for all parameters (Table 5).

Table 3: Frequency of the scores in the $4^{\text {th }}$ week.

\begin{tabular}{|lllllll|}
\hline & Erythema & & Pruritis & & Scaling \\
& Group A & Group B & Group A & Group B & Group A & Group B \\
\hline 0 (absent) & 16 & 24 & 11 & 17 & 15 & 25 \\
\hline 1 (mild) & 11 & 7 & 14 & 14 & 12 & 5 \\
\hline 2 (moderate) & 0 & 0 & 2 & 0 & 0 & 1 \\
\hline 3 (severe) & 0 & 0 & 0 & 0 & 0 & 0 \\
\hline
\end{tabular}

Table 4: Mean Scores $( \pm \mathrm{SD})$ for Group A.

\begin{tabular}{|llllll|}
\hline & Baseline (week 1) & Week 2 & Week 3 & Week 4 & P value \\
\hline Erythema & $1.75 \pm 0.80$ & $1.18 \pm 0.72$ & $0.81 \pm 0.62$ & $0.41 \pm 0.50^{*}$ & 0.0001 \\
\hline Pruritis & $1.43 \pm 0.84$ & $0.96 \pm 0.79$ & $0.78 \pm 0.70$ & $0.67 \pm 0.62^{*}$ & 0.0004 \\
\hline Scaling & $2.23 \pm 0.82$ & $1.14 \pm 0.71$ & $0.74 \pm 0.66$ & $0.44 \pm 0.51^{*}$ & 0.0001 \\
\hline
\end{tabular}

Table 5: Mean Scores ( \pm SD) for Group B.

\begin{tabular}{|lllll|l|}
\hline & Baseline $($ week 1$)$ & Week 2 & Week 3 & Week 4 & P value \\
\hline Erythema & $1.75 \pm 0.72$ & $1.16 \pm 0.68$ & $0.88 \pm 0.55$ & $0.23 \pm 0.43 *$ & 0.0001 \\
\hline Pruritis & $1.88 \pm 0.75$ & $1.47 \pm 0.84$ & $0.84 \pm 0.63$ & $0.45 \pm 0.51^{*}$ & 0.0001 \\
\hline Scaling & $2.06 \pm 0.84$ & $1.19 \pm 0.74$ & $0.72 \pm 0.77$ & $0.23 \pm 0.50^{*}$ & 0.0001 \\
\hline
\end{tabular}

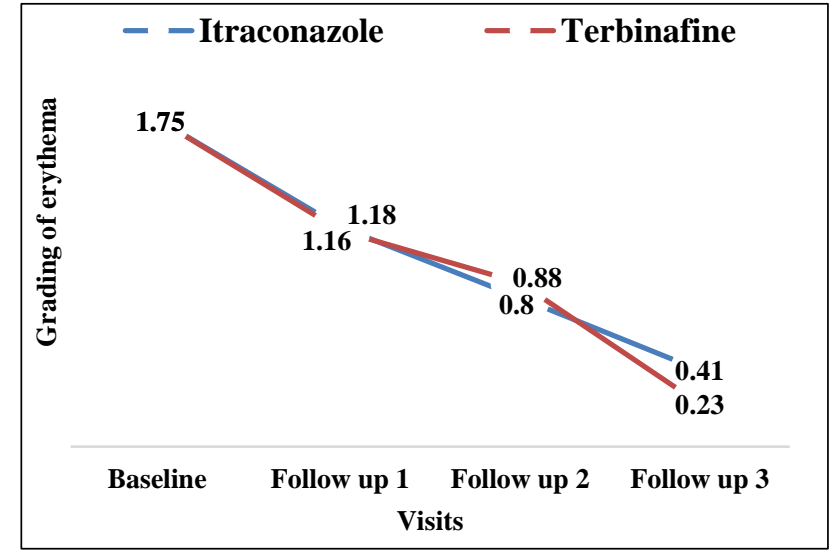

Figure 2: Comparison of erythema scores for the two medications.

The mean erythema scores of the two drugs were nearly equal for the first three visits but during the final visits $\left(4^{\text {th }}\right.$ week), erythema score for group A was 0.41 while that of group B was 0.23. Overall, differences at week 4 from the baseline scores for groups A and B were 1.34 and 1.52 respectively (Figure 2 ).

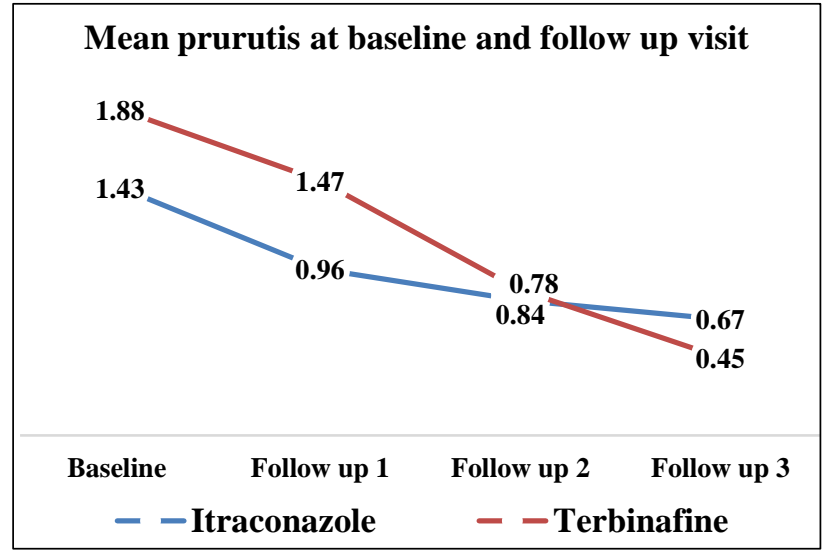

Figure 3: Comparison of pruritis scores for the two medications.

Initially, the mean pruritis score for group A (Itraconazole) was 1.43 and group B was 1.88. The score for group A decreased dramatically during the second week (with a difference of 1.07) whereas group B showed a difference of only 0.40 in that week. 
The scores decreased progressively for the two drugs for the next two weeks and finally at week 4, the scores for Group A and B were 0.67 and 0.45 respectively. Group B showed an overall difference of 1.43 while group A showed only a difference of 0.76 from the baseline visit (Figure 3).

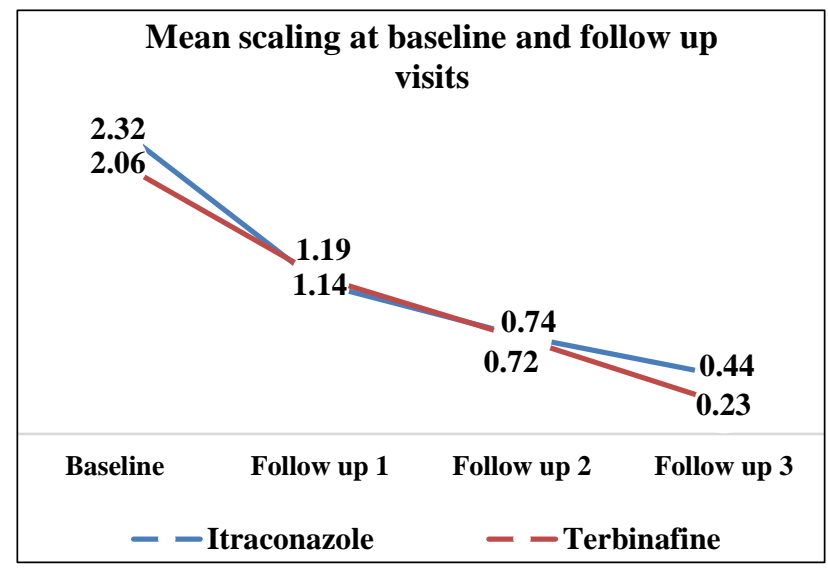

Figure 4: Comparison of scaling scores for the two medications.

The baseline scores for scaling were 2.23 for group A and 2.06 for Group B. At the end of the $4^{\text {th }}$ week, the differences in the scores were 1.79 for group A and 1.83 for group B (Figure 4).

The difference between the two groups for all 3 parameters were found to be statistically insignificant with $\mathrm{P}$ value being greater than 0.05 at week 4 from the baseline visit (Table 6).

Table 6: Comparison of the mean scores $( \pm \mathrm{SD})$ of Group $A$ and B at 4 weeks from baseline visit.

\begin{tabular}{|llll|}
\hline & Group A & Group B & P value \\
\hline Erythema & $0.41 \pm 0.50$ & $0.23 \pm 0.43$ & 0.1460 \\
\hline Pruritis & $0.67 \pm 0.62$ & $0.45 \pm 0.51$ & 0.1438 \\
\hline Scaling & $0.44 \pm 0.51$ & $0.23 \pm 0.50$ & 0.1196 \\
\hline
\end{tabular}

\section{DISCUSSION}

Dermatophytosis is prevalent on a global scale, accounting for $20-25 \%$ of the world's superficial mycotic infections. ${ }^{15}$ In India, where the environmental and demographic factors widely influence the occurrence of tinea cruris, it would be beneficial to evaluate the commonly used medications for this infection.

There were 9 diabetic patients who participated in this study. Diabetes, being a state of immune deficiency, is considered to be an important predisposing factor for tinea. Hyperglycaemia, particularly uncontrolled hyperglycaemia, aids the development of dermatophytosis and bacterial infections because such patients often have impaired cellular immunity and reduced phagocytic function of polymorphonuclear leucocytes. ${ }^{16}$ To better appreciate potential multidrug interactions and ensure their safety, patients' hypertensive histories were also noted. ${ }^{17}$ There were $97 \%$ of the patients who participated in this study belonged to lower socio-economic class and these patients were particularly susceptible to mycoses because of their poor personal hygiene habits and lack of proper environmental sanitation; transmission of this infection occurring via infected persons and fomites. ${ }^{18}$

Overall, the drugs were well tolerated, and patient compliance was fairly good (nearly 97\%). There were initially 60 patients included in the study. 2 male patients dropped out of this study during the second week while all female patients completed their therapy. There is a rising prevalence of ringworm among Indians due to the hot and humid climate as well as the poor hygienic practices; all of which contribute excessive sweating. Since sweat does contain water, salt, sugar and nitrogenous compounds, it is an ideal culture medium for these fungi. ${ }^{19}$ Majority of the female patients in the study ( 24 of them, more specifically) were homemakers who spend hours perspiring in the kitchen, while cooking. Remaining 5 females claimed to have been in contact with either friends or family members who suffered from the same infection as well. Male patients, however, had varied occupations, ranging from laborers and tailors to teachers and businessmen. A shared aspect among all these patients was the environment in which they were working. All of them complained of sweating profusely due to long working hours in the sun or in extremely humid locations which did not provide them with the luxury of an air conditioner (or even fans in certain cases).

In this study, authors found Terbinafine (group B) to be slightly more efficacious than Itraconazole (group A), prominently seen in the third week of the treatment (Table 2,3). After taking initial scores of the three parameters: erythema, pruritis and scaling during the baseline visits to the hospital, they were compared to the scores obtained in the next three weeks. The mean scores of the two drugs were similar for the first three visits. During the final visits (in the $4^{\text {th }}$ week), the difference in the scores for terbinafine were slightly higher than those of Itraconazole but after analysis, this difference was found to be statistically insignificant.

The findings of this study were consistent with that of a long-term study conducted in Europe which showed that Terbinafine $250 \mathrm{mg}$ per day for 12 weeks showed better mycological and cure rates for toenail onychomycosis than intermittent Itraconazole (400 mg a day a week for 12 weeks). Cure rates Terbinafine improved clearly and consistently until the 72 weekend point while Itraconazole showed no significant improvement after week $48 .{ }^{20}$ In a similar randomized, controlled trial, 4 weeks of treatment with oral administration of Terbinafine demonstrated higher overall efficacy rates $(100 \%)$ and lower relapse rates $(16 \%)$ as compared with Itraconazole (89\% efficacy, $75 \%$ relapse). ${ }^{21}$ 
Such differences in the scores can be attributed to the fungicidal action of Terbinafine. It abates the formation of ergosterol by inhibiting squalene epoxidase, the catalytic enzyme responsible for converting squalene to 2, 3oxidosqualene (an ergosterol precursor). The resultant deficiency in ergosterol compromises cell wall integrity and contributes to impaired growth and/or death of the pathogen. ${ }^{22}$ Terbinafine is the only commercially available allylamine that can be administered orally. On the other hand, Itraconazole is a broad spectrum anti-fungal with a fungistatic action; it inhibits cytochrome P450-dependent synthesis of ergosterol (a vital component of fungal cell membranes). ${ }^{23}$ Sertaconazole was also added to the treatment because of its fungicidal action. With its unique benzothiophene ring, Sertaconazole is the only azole that is capable of causing direct damage to the fungal cell membrane. This benzothiophene ring is also lipophilic in nature, enabling prolonged dermal retention that permits just once-daily application of $2 \%$ sertaconazole cream. ${ }^{4}$

Authors have examined and graded the signs of the infection and have correlated the resulting data to various socio-demographic and clinical variables. A basic literature search reveals that previously other well-known azoles (Miconazole, Ketoconazole) have been collectively compared with Terbinafine in the treatment of primarily onychomycosis. Not many studies, which compare only Itraconazole with terbinafine in the treatment of tinea cruris, have been conducted. This study was the first of its kind at this tertiary care hospital, comparing only Itraconazole to Terbinafine for tinea cruris, both of which were administered systemically.

\section{CONCLUSION}

Demographic factors and immune status of the patients were important predictors of tinea cruris. There were significant improvements in the symptoms when using both Terbinafine and Itraconazole. Although Terbinafine displayed a higher efficacy than Itraconazole, the difference between the mean scores of the two drugs was found to be statistically insignificant. Limitations of this study included its small sample size and short duration of follow-ups. Due to short duration of the follow-ups, we could not evaluate patients' relapse rates. Since was conducted at a single center, these findings cannot be generalized. For this purpose, further multicenter studies with larger sample size are required.

\section{ACKNOWLEDGEMENTS}

Authors would like to thank Dean Dr Pankaj Patel and all the patients who participated in the study.

Funding: No funding sources Conflict of interest: None declared

Ethical approval: The study was approved by the Institutional Ethics Committee

\section{REFERENCES}

1. Pires CA, Cruz NF, Lobato AM, Sousa PO, Carneiro FR, Mendes AM. Clinical, epidemiological, and therapeutic profile of dermatophytosis. An Bras Dermatol. 2014;89(2):259-64.

2. Seebacher C, Bouchara JP, Mignon B. Updates on the epidemiology of dermatophyte infections. Mycopathologia. 2008;166(5-6):335.

3. Naglot A, Shrimali DD, Nath BK, Gogoi HK, Veer V, Chander J, et al. Recent Trends of Dermatophytosis in Northeast India (Assam) and Interpretation with Published Studies. Int J Curr Microbiol Applied Sci. 2015;4(11):111-20.

4. Chatterjee D, Ghosh SK, Sen S, Sarkar S, Hazra A, De R. Efficacy and tolerability of topical sertaconazole versus topical terbinafine in localized dermatophytosis: A randomized, observer-blind, parallel group study. Indian J Pharmacol. 2016;48(6):659-64.

5. Sahoo AK, Mahajan R. Management of tinea corporis, tinea cruris, and tinea pedis: A comprehensive review. Indian Dermatol Online J. 2016;7(2):77-86.

6. Rajagopalan M, Inamadar A, Mittal A, Miskeen AK, Srinivas CR, Sardana K, et al. Expert Consensus on the Management of Dermatophytosis in India (ECTODERM India). BMC Dermatol. 2018;18(1):6.

7. Clinard VB, Smith JD. Cutaneous Fungal Infections. US Pharm. 2015;40(4):35-9.

8. Chatterjee D, Ghosh SK, Sen S, Sarkar S, Hazra A, De R. Efficacy and Tolerability of Topical Sertaconazole versus Topical Terbinafine in Localized Dermatophytosis: A Randomized, Observer-blind, Parallel Group Study. Indian J Pharmacol. 2016;48(6):659-64.

9. Ogba OM, Abia-Bassey LN. Tinea Cruris Resurgence in Male Genitalia: A Case Report. Int J Basic Applied Innovative Res. 2013;2(3):51-4.

10. Sheehan DJ, Hitchcock CA, Sibley CM. Current and Emerging Azole Antifungal Agents. Clin Microbiol Rev. 1999;12(1):40-79.

11. Jerajani HR, Janaki C, Kumar S, Phiske M. Comparative assessment of the efficacy and safety of sertaconazole $(2 \%)$ cream versus terbinafine cream $(1 \%)$ versus luliconazole $(1 \%)$ cream in patients with dermatophytoses: A pilot study. Indian J Dermatol. 2013;58(1):34-8.

12. Abdel-Rahman SM, Nahata MC. Oral terbinafine: a new antifungal agent. Ann Pharmacother. 1997;31(4):445-56.

13. Panagiotidou D, Kousidou T, Chaidemenos G, Karakatsanis G, Kalogeropoulou A, Teknetzis A, et al. A comparison of itraconazole and griseofulvin in the treatment of tinea corporis and tinea cruris: a doubleblind study. J Int Med Res. 1992;20(5):392-400.

14. Elman S, Hynan LS, Gabriel V, Mayo MJ. The 5-D Itch Scale: A New Measure of Pruritus. Brit J Dermatol. 2010;162(3):587-93. 
15. Havlickova B, Czaika VA, Friedrich M. Epidemiological trends in skin mycoses worldwide. Mycoses. 2009;52(1):95.

16. Akkus G, Evran M, Gungor D, Karakas M, Sert M, Tetiker T. Tinea pedis and onychomycosis frequency in diabetes mellitus patients and diabetic foot ulcers. A cross sectional-observational study. Pak J Med Sci. 2016;32(4):891-5.

17. Elewski B, Tavakkol A. Safety and tolerability of oral antifungal agents in the treatment of fungal nail disease: a proven reality. Therapeut Clin Risk Management. 2005;1(4):299-306.

18. Kundu D, Mandal L, Sen G. Prevalence of Tinea capitis in school going children in Kolkata, West Bengal. J Natural Sci Biol Med. 2012;3(2):152-5.

19. Levin OL, Silvers SH. The isolation of ringworm fungi from sweat. Arch Derm Syphilol. 1931;23(6):1094-9.

20. Evans EG, Sigurgeirsson B. Double blind, randomised study of continuous terbinafine compared with intermittent itraconazole in treatment of toenail onychomycosis. The LION Study Group. BMJ. 1999;318(7190):1031-5.
21. Budimulja U, Kuswadji K, Bramono S, Basuki J, Judanarso LS, Untung S, et al. A double-blind, randomized, stratified controlled study of the treatment of tinea imbricata with oral terbinafine or itraconazole. Br J Dermatol. 1994;130(43):29-31

22. Newland JG, Abdel-Rahman SM. Update on terbinafine with a focus on dermatophytoses. Clin Cosmetic Invest Dermatol. 2009;2:49-63.

23. Itraconazole FDA Label-Capsule. National Institutes of Health, U.S. Department of Health and Human Services. Available at: aidsinfo.nih.gov/drugs/44/itraconazole/59/profession al.

Cite this article as: George M, Chaudhary RG, Rana D, Kasundra D, Chaudhary AR, Malhotra SD. Comparative evaluation of efficacy of terbinafine and itraconazole in treatment of tinea cruris. Int $\mathbf{J}$ Basic Clin Pharmacol 2019;8:1460-6. 